This item was submitted to Loughborough's Research Repository by the author.

Items in Figshare are protected by copyright, with all rights reserved, unless otherwise indicated.

\title{
How predation can slow, stop or reverse a prey invasion
}

PLEASE CITE THE PUBLISHED VERSION

LICENCE

CC BY-NC-ND 4.0

\section{REPOSITORY RECORD}

Owen, Markus R., and M.A. Lewis. 2019. "How Predation Can Slow, Stop or Reverse a Prey Invasion". figshare. https://hdl.handle.net/2134/763. 


\title{
How predation can slow, stop or reverse a prey invasion
}

\author{
M. R. OWEN \\ Nonlinear and Complex Systems Group, \\ Department of Mathematical Sciences, \\ Loughborough University, \\ Loughborough, LE11 3TU, UK \\ E-mail: M.R.Owen@lboro.ac.uk
}

\author{
M. A. LEWIS \\ Department of Mathematics, \\ University of Utah, \\ Salt Lake City, \\ Utah 84112, USA \\ E-mail: mlewis@math.utah.edu
}

Key words: Predator-prey, Primary succession, Mount St. Helens

\begin{abstract}
Observations on Mount St. Helens indicate that the spread of recolonising lupin plants has been slowed due to the presence of insect herbivores and it is possible that the spread of lupins could be reversed in the future by intense insect herbivory (Fagan and Bishop, 2000). In this paper we investigate mechanisms by which herbivory can contain the spatial spread of recolonizing plants. Our approach is to analyse a series of predator-prey reaction-diffusion models and spatially coupled ordinary differential equation models to derive conditions under which predation pressure can slow, stall or reverse a spatial invasion of prey. We focus on models where prey disperse more slowly than predators. We comment on the types of functional response which give such solutions, and the circumstances under which the models are appropriate.
\end{abstract}

(c) 2000 Society for Mathematical Biology

\section{Introduction}

This study is motivated by interesting data on the recolonisation by lupins (Lupinus lepidus) of Mount St. Helens' north slope. The eruption of Mount St. Helens in 1980 caused complete extermination of all plant and animal species in a large area known as the Pumice Plains, presenting an excellent opportunity for the study of primary succession - the formation of biological communities in the absence of historical influences.

In 1981, a species of lupin began to recolonize the Pumice Plains region of Mount St. Helens. In the mid 1980s, the first herbivore populations (chiefly 0092-8240/00/010001 + 35 \$17.00/0mb00????C) 2000 Society for Mathematical Biology 
lupin-specific lepidopterans) were identified within the lupin colonies, and in 1990 these herbivores first reached the lupin 'wavefront' (Fagan and Bishop, 2000) which was several kilometres away from the initial introduction site. Experiments at the site show that the herbivores induce a decrease in the per capita growth rate of lupins at low densities, and it has been suggested that these herbivores may stall or even reverse the spread of lupins (Fagan and Bishop, 2000). This leads to the question of whether such behaviour is possible in models for predator-prey dynamics, where the herbivore is the 'predator' and the lupin is the 'prey'.

In keeping with the biology we assume that the prey disperse much more slowly that the predators. Our main focus is analysis of spread rates for predator and prey populations in nonlinear reaction-diffusion models. First we consider simple predator-prey models, described by convex nonlinear growth of prey, types I-III functional and numerical responses, and diffusion of prey and predator (Section 2). We demonstrate that the introduction of predators into such systems will not reduce prey spread rate. We then move to a more general model framework for predator-prey models which includes nonconvex growth functions for the prey. We demonstrate that to slow population spread via predation requires at least a 'weak' Allee effect for the prey-only dynamics (reduced growth rate for unexploited prey population at low density), and to reverse population spread via predation requires a 'strong' Allee effect for the prey-only dynamics (negative growth rates for unexploited prey populations when at low density). In the reaction-diffusion formulations, stationary (zero spread rate) solutions are structurally unstable. However, this is not the case for spatial patch models. In Section 3 we consider an explicitly patchy model, given by spatially coupled ODEs, which admits stationary solutions for a range of parameter values. Section 4 includes a discussion, together with suggestions for future research.

\section{A reaction-diffusion predator-prey model}

We consider the nonlinear reaction-diffusion predator-prey model

$$
\begin{aligned}
& u_{t}=\varepsilon D u_{x x}+r u f(u)-\phi v h(u) \\
& v_{t}=D v_{x x}+\gamma v h(u)-\delta v
\end{aligned}
$$

This model arises from adding spatial movement terms, described with diffusion, to a classical predator-prey dynamics (see, for example, May (1974)). The per capita prey growth rate is $r f(u)$. The strictly monotonic functional and numerical responses, $\phi h(u)$ and $\gamma h(u)$ have identical form, with $\gamma / \phi$ describing conversion efficiency. We define $K$ to be the carrying capacity for the prey in the absence of predation and we assume that, in the absence 
of prey, predator populations will not grow, so that $f(K)=h(0)=0$. We also assume that the model has a coexistence equilibrium $\left(u_{s}, v_{s}\right)$ which is stable in the ODE sense (i.e., in the absence of diffusion). The parameters $r$ and $\phi$ allow us to scale the continuous functions $f$ and $h$ so that $\max _{u \in[0, K]} f(u)=h(K)=1$. The parameter $\varepsilon, 0<\varepsilon \ll 1$, reflects the assumption that prey disperses much more slowly than predators.

Introducing the following dimensionless quantities:

$$
\begin{gathered}
t^{*}=r t, \quad x^{*}=x \sqrt{\frac{r}{D}}, \quad u^{*}=u / K, \quad v^{*}=\frac{\phi v}{r K}, \\
f^{*}\left(u^{*}\right)=f(u), \quad h^{*}\left(u^{*}\right)=h(u), \quad \gamma^{*}=\frac{\gamma}{r}, \quad \delta^{*}=\frac{\delta}{\gamma},
\end{gathered}
$$

and dropping asterisks for notational simplicity gives

$$
\begin{aligned}
& u_{t}=\varepsilon u_{x x}+u\left(f(u)-\frac{v}{u} h(u)\right) \\
& v_{t}=v_{x x}+\gamma v(h(u)-\delta)
\end{aligned}
$$

Our conditions on $f$ and $h$ become:

$$
f(1)=0, \quad \max _{u \in[0,1]} f(u)=1, \quad h(0)=0, \quad h(1)=1 .
$$

Steady states for this model are an extinction steady state, $(u, v)=(0,0)$, a prey-only steady state $(u, v)=(1,0)$ - corresponding to primary succession before predators have arrived, and a coexistence steady state

$$
u_{s}=h^{-1}(\delta), \quad v_{s}=\frac{u_{s} f\left(u_{s}\right)}{h\left(u_{s}\right)}=\frac{u_{s} f\left(u_{s}\right)}{\delta} .
$$

The relevant range of predator mortality rate $\delta$ for a nonnegative coexistence equilibrium is $0 \leq \delta \leq 1$.

Stability of the prey carrying capacity in the absence of predation and stability of the coexistence equilibrium require that

$$
f^{\prime}(1)<0, \quad g^{\prime}\left(u_{s}\right)<0,
$$

where

$$
g(u)=u \frac{f(u)}{h(u)} .
$$

Because $h$ is strictly monotonic with root at $u=0, g$ is defined for positive $u$.

Extensive simulations of the full nonlinear PDE model with zero flux boundary conditions indicate that compact initial data $u(x, 0)$ and $v(x, 0)$ 
numerically converge to travelling wave solutions for a wide variety of growth functions $f$ and functional responses $h$, providing $\varepsilon$ is sufficiently small, i.e., providing the predators diffuse fast enough relative to prey so as to be able to 'catch up' with the prey. An example, shown in Figure 1, uses logistic growth for the prey and type II predation.

In Figure 1 the predators catch up to the prey, but do not slow the spread of prey at all. In Section 2.2 we will show that this is a general feature of predator-prey models with logistic prey growth. However, as we will show in Section 2.3, introduction of an Allee effect in the prey dynamics makes it possible for the spreading prey to be slowed by predation. Another kind of solution is also possible, with or without an Allee effect for the prey. Predators can stop the prey as they approach the right hand end of the domain. This case has been used by Hastings and coworkers (Hastings et al., 1997) to explain stationary predator-prey distributions. However, in the context of predator-prey invasions, we will show that existence of this stationary solution depends upon zero-flux boundary conditions for predators and prey (see Section 2.4).
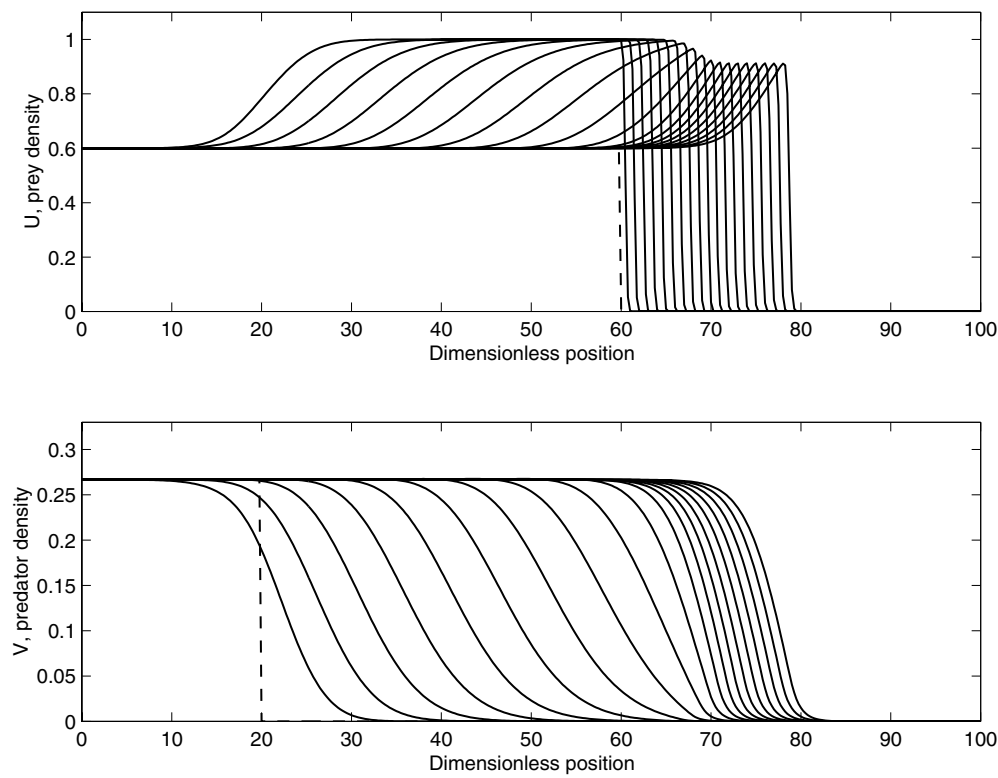

Figure 1. With logistic growth $f(u)=1-u$ and type II predation $h(u)=$ $(\alpha+1) u /(\alpha+u)$, introduced predators catch up to prey and form a travelling wave joining extinction and coexistence equilibria. Notice that the predators do not slow the spread of prey after they catch up with them. Boundary conditions are zero flux for $u$ and $v$. Parameter values are $\delta=0.9, \alpha=0.2, \gamma=1, \varepsilon=0.001$ in (3). Dashed lines show initial conditions, solid lines show solutions up to $t=200$ at intervals of 10 dimensionless time units.

We know of no proof of existence and convergence of initial data to trav- 
elling waves such as those seen in Figure 1. This remains an interesting open problem. Work by Dunbar (1986) proves existence for similar dynamics, but with boundary conditions describing spread of predators into prey. Also, related work by Sherratt et al. (1997) and others shows that predators spreading into prey can exhibit complex spatiotemporal behaviour which cannot be described by a travelling wave if the coexistence equilibrium is unstable, i.e., if the second condition in (5) is violated. However, based on our numerical results, we consider the case where compact initial data for (3) has converged to an expanding wave solution whose spread, both to the left and to the right, asymptotically takes the form of a travelling wave. Without loss of generality we consider the travelling wave front connecting $\left(u_{s}, v_{s}\right)$ with $(0,0)$ :

$$
\begin{gathered}
0=c U^{\prime}+\varepsilon U^{\prime \prime}+U\left(f(U)-\frac{V}{U} h(U)\right) \\
0=c V^{\prime}+V^{\prime \prime}+\gamma v(h(U)-\delta) . \\
\lim _{z \rightarrow \infty} U(z)=\lim _{z \rightarrow \infty} V(z)=0, \quad \lim _{z \rightarrow \infty} U(z)=u_{s}, \quad \lim _{z \rightarrow \infty} V(z)=v_{s}
\end{gathered}
$$

where $z=x-c t$ is the travelling wave coordinate with wave velocity $c$.

2.1. Prey-only waves. Existence of travelling waves and convergence of initial data to such waves have been analysed in detail for the prey-only model

$$
u_{t}^{+}=\varepsilon u_{x x}^{+}+u^{+} f\left(u^{+}\right) .
$$

by Aronson and Weinberger (1975) and others. Providing $u^{+} f\left(u^{+}\right)$has the appropriate concave-down shape

$$
f(u)>0 \quad \text { on } \quad 0<u<1, \quad f(u)<0 \quad \text { for } \quad u>1, \quad f(0)=1
$$

then compact initial data converge asymptotically to left- and right-moving travelling waves with speed

$$
c^{+}=2 \sqrt{\varepsilon f(0)} .
$$

Without loss of generality we focus on the right-moving wave. This joins $u=1$ and $u=0$ with a monotonic wave $u^{+}(x, t)=U^{+}\left(x-c^{+} t\right)$ with velocity $c^{+}$and $0 \leq U^{+} \leq 1$.

The above conditions on $f(10)$ are sufficient to guarantee wave speed (11) for equation (9), but are not necessary. In particular, the condition that the maximum per capita growth rate occurs at the lowest possible density 
$(f(0)=1)$ can sometimes be relaxed. By way of example, if we choose the quadratic function

$$
f=k(1-u)(u-a), \quad a \leq 1
$$

with the scaling factor $k$ so that the maximum per capita growth rate is unity,

$$
k=\left\{\begin{array}{ll}
-1 / a & \text { for } a \leq-1 \\
4 /(1-a)^{2} & \text { for }-1 \leq a<1
\end{array},\right.
$$

then spread rate for $(9)$ is

$$
c^{+}= \begin{cases}2 \sqrt{-\varepsilon a k} & \text { for } a \leq-1 / 2 \\ \sqrt{2 \varepsilon k}(1 / 2-a) & \text { for }-1 / 2 \leq a \leq 1\end{cases}
$$

(Hadeler and Rothe, 1975; Rothe, 1981; Lewis and Kareiva, 1993). The sufficient conditions given above (10) guarantee wave speed $c^{+}=2 \sqrt{-\varepsilon a k}$ only for the values of $a$ yielding $f(0)=1,(a \leq-1)$ whereas this speed is also correct for $-1<a \leq-1 / 2$. We will use this quadratic $f$ given in equation (12) in an example below.

Waves with speed given by (11) are called 'pulled waves' (Hadeler and Rothe, 1975) because the speed is governed by behaviour of the leading edge. Linearizing about $u^{+}=0$ yields

$$
u_{t}^{+}=\varepsilon u_{x x}^{+}+u^{+} f(0),
$$

and, for compact initial data, it is straightforward to show that the spread rate for any level set $u=u_{L}$ asymptotically achieves (11) (Aronson and Weinberger, 1975). Waves that are not pulled are said to be 'pushed' (Hadeler and Rothe, 1975). Here, the dynamics exhibit an Allee effect and population growth at intermediate densities drives the wave forward as individuals reproduce at high rates and spill over, via diffusion, to the front of the wave where per capita growth rates are lower. Thus sufficient conditions for a pulled wave are given by (10), although these may not be necessary.

2.2. Linear analysis. In this section we show that introduction of predators will not slow the spread of prey, providing the prey-only wave (9) is a 'pulled wave', i.e., has speed $c^{+}$given by (11) (see above). We show this by demonstrating that the predator-prey travelling wave can move no slower than $c^{+}(11)$.

It is straightforward to show that, in the absence of diffusive terms, the region $0 \leq u \leq 1, v \geq 0$ is an invariant set for (3). It follows that this region remains invariant when the diffusion terms are included (Smoller, 1994). Thus nonnegative initial data will remain nonnegative for all time 
$t$ and space $x$ values, and prey density will remain below or at carrying capacity $(u=1)$ for all time and space, providing it initially starts below or at carrying capacity. Furthermore, if the compact initial prey density $u(x, 0)$ in $(3)$ is above carrying capacity, we can bound $u(x, t)$ above for all time $t>0$ by the solution $u^{+}(x, t)$ to the model with no predation (9) and initial data $u(x, 0)$. As described above this solution $u^{+}(x, t)$ asymptotically lies between zero and one, and we thus conclude the same for $u(x, t)$.

We now use the above results coupled with linear analysis to show that predator-prey travelling waves can go no slower than $c^{+}(11)$. In some neighbourhood of any hyperbolic equilibrium $(u, v)=(\hat{u}, \hat{v})$ for $(7)$, flow of the nonlinear system is governed by its linearisation,

$$
\begin{aligned}
& 0=c U^{\prime}+\varepsilon U^{\prime \prime}+\left(\hat{u} f^{\prime}(\hat{u})+f(\hat{u})-\hat{v} h^{\prime}(\hat{u})\right) U-h(\hat{u}) V \\
& 0=c V^{\prime}+V^{\prime \prime}+\gamma \hat{v} h^{\prime}(\hat{u}) U+\gamma(h(\hat{u})-\delta) V
\end{aligned}
$$

(Perko, 1991). Eigenvalues $\lambda$ for this linear system satisfy

$$
\left|\begin{array}{cc}
\varepsilon \lambda^{2}+c \lambda+\hat{u} f^{\prime}(\hat{u})+f(\hat{u})-\hat{v} h^{\prime}(\hat{u}) & -h(\hat{u}) \\
\gamma \hat{v} h^{\prime}(\hat{u}) & \lambda^{2}+c \lambda+\gamma(h(\hat{u})-\delta)
\end{array}\right|=0 .
$$

Analysis about the leading edge of the wave $(\hat{v}=0)$ yields

$$
\lambda_{ \pm}^{U}=\frac{-c \pm \sqrt{c^{2}-4 \varepsilon\left(\hat{u} f^{\prime}(\hat{u})+f(\hat{u})\right)}}{2 \varepsilon},
$$

with eigenvector $\left(U, U^{\prime}, V, V^{\prime}\right)=\left(1, \lambda_{ \pm}^{U}, 0,0\right)$, and

$$
\lambda_{ \pm}^{V}=\frac{-c \pm \sqrt{c^{2}-4 \gamma(h(\hat{u})-\delta)}}{2}
$$

with eigenvector $\left(U, U^{\prime}, V, V^{\prime}\right)=\left(0,0,1, \lambda_{ \pm}^{V}\right)$. Thus, analysis about the leading edge of the travelling wave $(7)-(8), \hat{u}=0$, gives a necessary condition

$$
c^{2} \geq 4 \varepsilon f(0)
$$

for nonnegative prey density.

Recall that when the prey-only wave is 'pulled' equation (11) gives its spread rate. In this case, we conclude from (20) that the predator-prey travelling wave moves no slower than the prey-only wave. Indeed, using the fact that the solution $u^{+}(x, t)$ to $(9)$ with initial data $u(x, 0)$ is an upper bound to the solution $u(x, t)$ to (3a) we observe that the predator-prey travelling wave must move at precisely the pulled speed so that $c=c^{+}=$ $2 \sqrt{\varepsilon f(0)}$. In addition, when $f$ satisfies the above sufficient condition for a 
pulled wave (10), then the predator-prey travelling wave has speed $c=c^{+}=$ $2 \sqrt{\varepsilon}$.

It is also instructive to consider the case where predators spread into a prey population which is at carrying capacity so that boundary conditions are

$$
\lim _{z \rightarrow \infty} U(z)=1, \quad \lim _{z \rightarrow \infty} V(z)=0, \quad \lim _{z \rightarrow-\infty} U(z)=u_{s}, \lim _{z \rightarrow-\infty} V(z)=v_{s}
$$

(see also Dunbar (1986)). Since $h(1)=1>\delta$, a necessary condition for predator density to remain nonnegative is

$$
c^{2} \geq 4 \gamma(1-\delta)
$$

Indeed, numerical simulations indicate that the speed that predators 'catch up' to a spreading prey population is given by smallest possible value of $c$ in (22). For example, the simulation illustrated in Figure 1 used $\varepsilon=0.001, \delta=$ $0.9, \gamma=1$, which gives predicted speeds for the predator-prey invasion as $c=0.063$ and for the predator catch-up wave as $c=0.63$. These match very closely with speeds calculated from the simulation speeds. Even though the minimum possible speed is often the one selected in multispecies waves (see for example, (Murray, 1989)), it should be noted that this is not always the case (Hosono, 1998). For further discussion of this see Weinberger et al. (2000a,b).

2.3. An example introducing the Allee effect. Our results from the previous section indicate that when the prey-only wave is a 'pulled wave' then the introduced predator cannot slow the wave, and prey and predator spread at the prey-only wave speed. If the predator is to stop the prey invasion, i.e. if $c=0$ in (7), equation (20) requires that $f(0) \leq 0$. In this case prey growth dynamics $u f(u)$ have a 'strong' Allee effect- the per capita prey growth rate must be negative at sufficiently small prey density. We refer to the case $0<f(0)<1$ as having a 'weak' Allee effect-the per capita prey growth rate is reduced but remains positive at small prey density. As shown below, the spread of prey populations with a weak Allee effect may be slowed but not stopped by introduction of the predator.

Figure 2 shows numerically calculated wave speeds for predator prey model (3), with $f$ given by (12) and a type I functional response $h(u)=u$. The solid and dashed curves are the first and second equations in (14), which determine the speed of the prey-only wave for this $f$. These curves are drawn heavier in their region of validity according to (14). The numerically calculated predator-prey wave speed matches the prey-only wave speed for values of $a$ where the prey-only wave is 'pulled' $(-2 \leq a \leq-0.5)$. It lies below the prey-only 'pushed' wave speed but on or above the linear wave speed for $-0.5 \leq a \leq 0.5$. 
Using the above terminology, the prey-only dynamics exhibit a weak Allee effect for $-1<a \leq 0$ and a strong Allee effect for $0<a \leq 0.5$. Thus the effect of introducing predators shows a wide range of behaviour. When the prey have a weak Allee effect $(-1<a \leq 0)$, the introduction of predators can $(-0.5 \leq a \leq 0)$, but need not $(-1 \leq a<-0.5)$ slow the spread of prey. When the prey have a strong Allee effect $(0<a \leq 0.5)$, the introduction of predators slows the prey and can $\left(a_{c}(\delta)<a \leq 0.5\right)$, but need not $\left(0<a \leq a_{c}(\delta)\right)$ reverse the spread of prey. Here the critical Allee threshold $a_{c}(\delta)$ for which predators can reverse prey spread depends upon predator mortality $\delta$. In this simulation $\delta=0.76$ and $a_{c}(\delta)=0.421$. As we will show in Section 2.6, $a_{c}(\delta) \in[0.394,0.5]$ for type I predation.

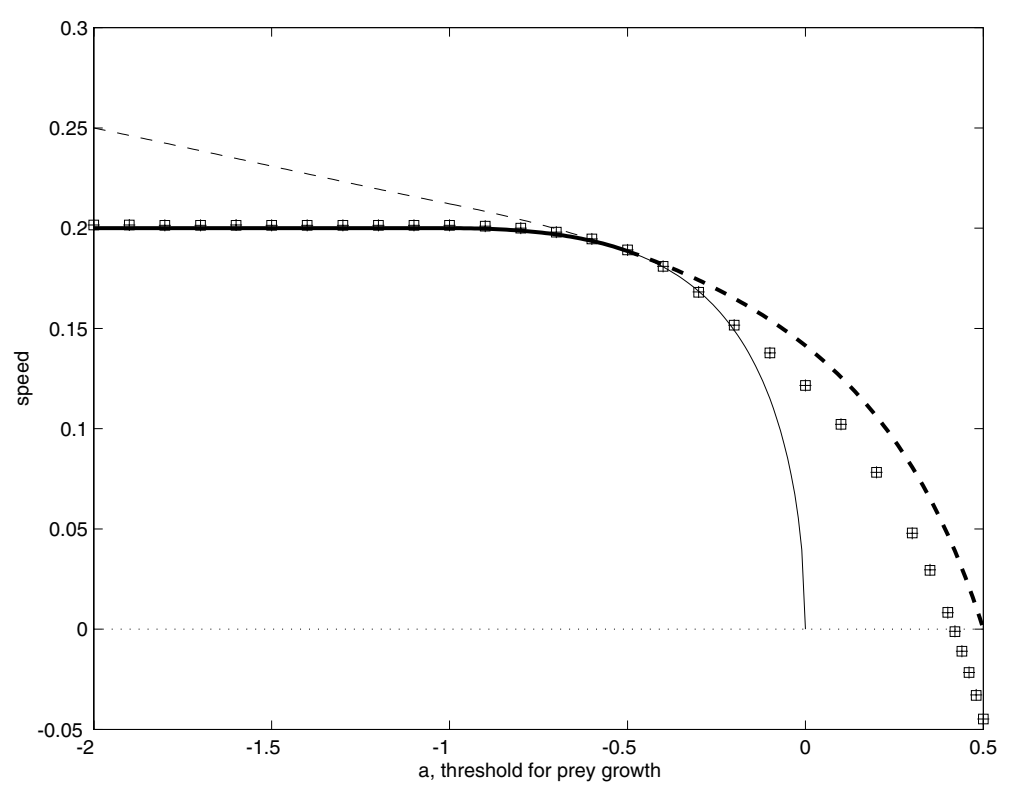

Figure 2. Numerical and theoretical wave speed results for a predator-prey system with cubic prey dynamics (3), (12) and type I predation $h(u)=u$. Model parameters are given by $\gamma=1, \delta=0.76$ and $\varepsilon=0.01$. Solid and dashed lines show the possible speeds for the prey-only case, given by the first and second equation of (14). Heavier lines indicate the speed appropriate for $a$ value, as indicated in (14). Crosses and squares show the speed of spread of both prey and predator.

Figure 3 shows the temporal progression for a simulation with an Allee effect in the prey dynamics: A small introduction of predators behind a colonising wave of prey rapidly catches up the prey wavefront and the two populations spread as a wave of coexistence. The speed of the wave of colonising prey is 0.07 , whereas that of the predator wave is 0.92 . Once the predators catch up to the colonising wave front, the speed of the resulting coexistence wave $(c=0.04)$ is less than that of the prey alone, and an order 
of magnitude less than that of the predators in their pursuit of the prey. Thus the predators have slowed the spread of the prey.
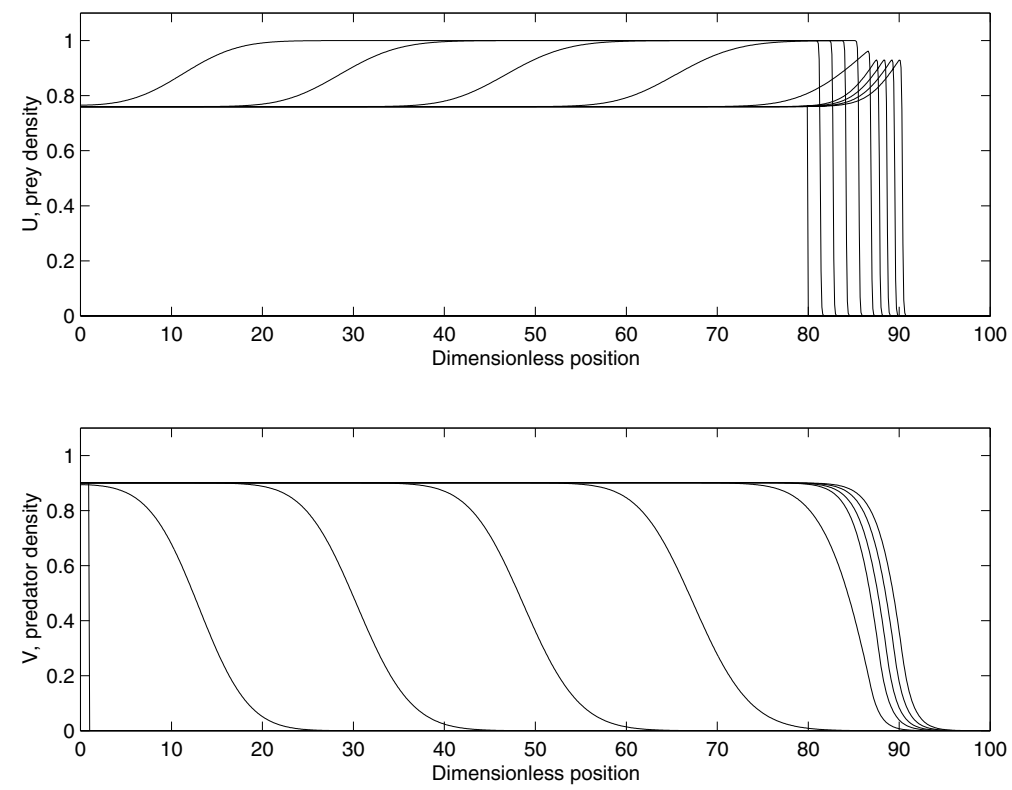

Figure 3. Spatial solution for the predator-prey model (3), with $f(u)=k(u-$ $a)(1-u)$, and $a=0.3$. Predators catching up colonising prey, and then slow the invasion. The dimensionless speed of the prey wave is 0.07 , and the speed of predator pursuit is 0.92 . Solutions are shown from $T=0$ to $T=200$ at intervals of 20 dimensionless time units. When the predators catch up the prey, the resulting coexistence wave moves at a speed of 0.04 . This slowing down can be seen in the prey profile, by observing that the gaps between successive wave fronts get shorter after the predators have caught up. Parameters are $\delta=0.76, \gamma=1, \varepsilon=0.01$ - these are the same as for the speed calculations for a range of $a$ as shown in Figure 2. Initial conditions, shown by the dashed lines, were of a prey population at its carrying capacity for $0 \leq x \leq 80$ and zero otherwise, and an introduction of predator density $v=0.9$ on $0 \leq x \leq 1$.

We now focus on the coexistence wavefront which arises after the predators have caught up with the prey. Because the movement of prey is small relative to predators $(\varepsilon=0.01)$ there is a sharp transition in $u$ from the coexistence steady state on the left to zero on the right. Inside this transition layer, $v$ is constant $\left(v=v_{0}\right)$ to leading order of $\varepsilon$. As discussed below, within the transition layer, $u$ is governed to leading order by a scalar PDE with $v=v_{0}$ as a parameter. Outside the transition layer, $\varepsilon u_{x x} \ll 1$, and so to leading order the prey population is determined by a first order ODE. Figure 4 shows the $(u, v)$ phase plane, including the nullclines, and with the final PDE solution from Figure 3 superimposed. As expected, trajectories follow the $u$-nullcline $v=g(u)$, until a rapid transition to the other nullcline $u=0$. This motivates the singular perturbation construction given below. 


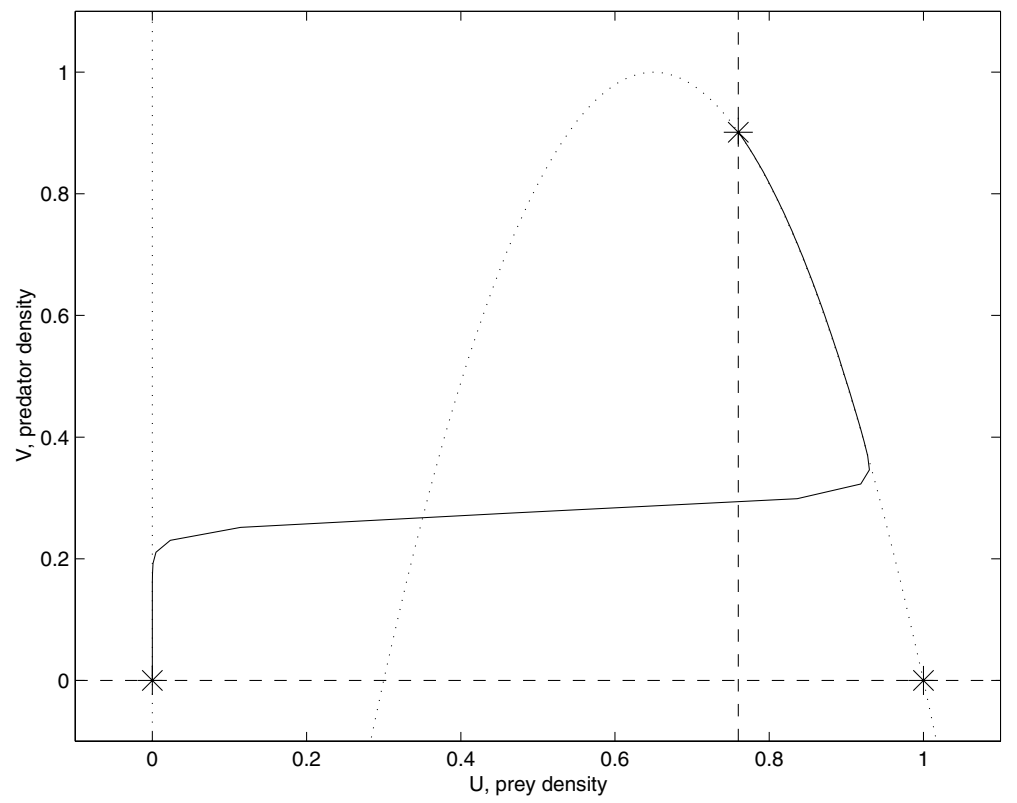

Figure 4. Predator-Prey phase plane for the model (3), with nullclines and PDE solutions. Steady states of the homogeneous system are marked with asterisks. The solutions clearly follow the $u$-nullclines (dotted lines), except for an abrupt transition between them. Model details and parameters are as in Figure 3. 
2.4. Singular perturbation analysis of the wave front. Our approach is to use the above ideas to formulate the travelling wave problem (7)-(8) in terms of transition and outer layers, and to concentrate on the construction of such solutions with zero speed $(c=0)$. This focus on stationary solutions is motivated by the idea that the boundary between invasive and recessive waves is given by the locus of points in parameter space that allows such solutions. We then extend this approach to cover the case where there is a stationary solution induced by zero-flux boundary conditions, henceforth referred to as an 'edge solution'.

Transition layer In the transition layer, $u$ varies rapidly while $v$ is, to a first approximation, constant. Rescaling space according to $x=\sqrt{\xi}$, stationary front solutions of (3) must satisfy

$$
\begin{aligned}
\frac{d^{2} u}{d \xi^{2}}+u\left(f(u)-v \frac{h(u)}{u}\right) & =0 \\
\frac{d^{2} v}{d \xi^{2}}+\varepsilon \gamma v(h(u)-\delta) & =0 .
\end{aligned}
$$

Now in the limit as $\varepsilon \rightarrow 0, v$ satisfies $\frac{d^{2} v}{d \xi^{2}}=0$, and since $v \geq 0$ as $\xi \rightarrow \pm \infty$, it follows that $v=v_{0}$, a constant to be determined. Thus, we have a single equation for $u$,

$$
\frac{d^{2} u}{d \xi^{2}}+u\left(f(u)-v_{0} \frac{h(u)}{u}\right)=0,
$$

together with boundary conditions:

$$
\begin{array}{r}
\lim _{\xi \rightarrow-\infty} u(\xi)=g^{-1}\left(v_{0}\right)=u_{0}, \lim _{\xi \rightarrow+\infty} u(\xi)=0, \\
\lim _{\xi \rightarrow-\infty} \frac{d u}{d \xi}(\xi)=0, \lim _{\xi \rightarrow+\infty} \frac{d u}{d \xi}(\xi)=0 .
\end{array}
$$

Here, $g^{-1}\left(v_{0}\right)$ implies the choice of the correct (descending through the coexistence steady state) branch of the $u$-nullcline $v=g(u)$. Multiplying the $u$ equation $(23)$ by $d u / d \xi$, noting that we have implicitly defined in the above boundary conditions that $v_{0}=g\left(u_{0}\right)$, and integrating with respect to $\xi$ yields

$$
\int_{-\infty}^{\infty}\left\{\frac{d^{2} u}{d \xi^{2}}+u\left(f(u)-g\left(u_{0}\right) \frac{h(u)}{u}\right)\right\} \frac{d u}{d \xi} d \xi=0 .
$$

The first term can be integrated directly, and for the second we use a change of variables from $\xi$ to $u$, to get

$$
\left[\frac{1}{2}\left(\frac{d u}{d \xi}\right)^{2}\right]_{-\infty}^{+\infty}+\int_{0}^{u_{0}} u\left(f(u)-g\left(u_{0}\right) \frac{h(u)}{u}\right) d u=0 .
$$


Applying the boundary conditions on $\frac{d u}{d \xi}$ gives the following equation which determines $u_{0}$ consistent with a stationary solution:

$$
\int_{0}^{u_{0}} u\left(f(u)-g\left(u_{0}\right) \frac{h(u)}{u}\right) d u=0 .
$$

Note that this analysis is equivalent to that for determining the direction of waves in the class of single variable PDEs with cubic-type kinetics (Murray, 1989). Clearly, to stand any chance of getting a stationary wave, the above integrand must have three zeros for some value of $u_{0}$. However, just determining a $u_{0}$ that satisfies (24) by no means guarantees a stationary solution, since we must be able to match this inner front with outer solutions. In particular, the outer solutions must attain $v_{0}=g\left(u_{0}\right)$ at the transition.

Right hand outer solutions Outside the transition layer, $u$ satisfies $u f(u)-$ $v h(u)=0$, or equivalently using (6) $u=0$ or $u=g^{-1}(v)$. We consider first the right hand outer solution, when $u=0$, so that for a stationary solution, $v$ must satisfy

$$
\frac{d^{2} v}{d x^{2}}-\gamma \delta v=0
$$

with boundary conditions

$$
\lim _{x \rightarrow+\infty} v(x)=0, v(0)=v_{0} .
$$

We use this boundary condition because we require outer solutions which give the zero speed value for the predator density, $v=v_{0}$, at the transition layer.

Equation (25) has general solution

$$
v(x)=A e^{\sqrt{\gamma \delta} x}+B e^{-\sqrt{\gamma \delta} x} .
$$

Thus, applying the boundary conditions, we have that $A=0$ and $B=v_{0}$, so that

$$
\frac{d v}{d x}(0)=-v_{0} \sqrt{\gamma \delta}
$$

This is used to calculate the left hand outer solution below.

Left hand outer solutions Now we consider the other outer layer. Recall that we are trying to construct outer solutions which match the inner solution at $v=v_{0}$. Conservation of flux across $v=v_{0}$ means that we must also match the derivative of $v$.

In the second outer layer we have

$$
\frac{d^{2} v}{d x^{2}}+\gamma v\left(h\left(g^{-1}(v)-\delta\right)=0\right.
$$


with boundary conditions:

$$
\begin{gathered}
v(-\infty)=v_{s}, \quad v(0)=v_{0}, \\
\frac{d v}{d x}(-\infty)=0, \quad \frac{d v}{d x}(0)=-v_{0} \sqrt{\gamma \delta},
\end{gathered}
$$

which enforces the conservation of flux described above. Multiplying by $d u / d x$ and integrating with respect to $x$ :

$$
\int_{-\infty}^{0}\left\{\frac{d^{2} v}{d x^{2}}+\gamma v\left(h\left(g^{-1}(v)-\delta\right)\right\} \frac{d v}{d x} d x=0 .\right.
$$

In the same way as the analysis in the transition layer, the first term can be directly integrated, and changing the variables from $x$ to $v$ gives

$$
\left[\frac{1}{2}\left(\frac{d v}{d x}\right)^{2}\right]_{-\infty}^{0}+\int_{v_{s}}^{v_{0}} \gamma v\left(h\left(g^{-1}(v)-\delta\right) d v=0 .\right.
$$

Thus,

$$
\int_{v_{0}}^{v_{s}} \gamma v h\left(g^{-1}(v)\right) d v=\frac{1}{2} \gamma \delta v_{0}^{2}-\int_{v_{s}}^{v_{0}} \gamma \delta v d v,
$$

and the left- and right-hand outer solutions match at $v_{0}$ if and only if

$$
\int_{v_{0}}^{v_{s}} v h\left(g^{-1}(v)\right) d v=\frac{\delta v_{s}^{2}}{2}
$$

This describes the relationship between nonlinear prey dynamics, $f$ and $h$ (recall $g(u)=u f(u) / h(u)$ ), and relative mortality rate of predators, $\delta$, which is necessary and sufficient for existence of a stationary solution, in the limit as $\varepsilon \rightarrow 0^{+}$.

Evaluating the left hand side of (29) can be awkward due to the necessity of defining $g^{-1}$, but fortunately a change of variables simplifies things considerably, and also gives a condition for $u_{0}=g^{-1}\left(v_{0}\right)$ directly. Setting $u=g^{-1}(v)$, it follows that $v=g(u)$ and $d v=g^{\prime}(u) d u$, thus

$$
\begin{aligned}
\int_{v_{0}}^{v_{s}} v h\left(g^{-1}(v)\right) d v & =\int_{g^{-1}\left(v_{0}\right)}^{g^{-1}\left(v_{s}\right)} g(u) h(u) g^{\prime}(u) d u \\
& =\int_{u_{0}}^{u_{s}} \frac{u f(u)}{h(u)} h(u) g^{\prime}(u) d u \\
& =\int_{u_{0}}^{u_{s}} u f(u) g^{\prime}(u) d u .
\end{aligned}
$$


For simplicity we also write the right hand side of (29) in terms of $u$,

$$
\frac{\delta v_{s}^{2}}{2}=\frac{\delta g\left(u_{s}\right)^{2}}{2}
$$

Finally, then, we collect together the two conditions which must be satisfied for $u_{0}$ and $\delta$ in order for zero wave speed solutions of (7)-(8) to exist in the limit as $\varepsilon \rightarrow 0^{+}$:

$$
\begin{gathered}
\int_{0}^{u_{0}} u\left(f(u)-g\left(u_{0}\right) \frac{h(u)}{u}\right) d u=0, \\
\int_{u_{0}}^{u_{s}} u f(u) g^{\prime}(u) d u-\frac{\delta g\left(u_{s}\right)^{2}}{2}=0 .
\end{gathered}
$$

Analysis on a semi-infinite domain We now consider a stationary solution to (7) on the semi-infinite domain $-\infty<z \leq 0$ with boundary conditions

$$
U^{\prime}(0)=0, \quad V^{\prime}(0)=0, \quad \lim _{z \rightarrow-\infty} U(z)=u_{s}, \quad \lim _{z \rightarrow-\infty} V(z)=v_{s}
$$

We assume that the transition layer for prey $U(z)$ is a jump from $U=u_{0}>0$ to $U=0$ at a predator level $V=v_{0}$ which occurs at location $z=-b<0$. Analysis similar to that given above yields (30) as a means of calculating $u_{0}$, and a modified version of (31)

$$
\int_{u_{0}}^{u_{s}} u f(u) g^{\prime}(u) d u-\frac{\delta g\left(u_{s}\right)^{2}}{2}=\frac{\delta g\left(u_{0}\right)^{2}}{2}\left(\tanh ^{2}(\sqrt{\gamma \delta} b)-1\right),
$$

from which $b$ can be calculated uniquely providing the left hand side of (33) lies between $-\delta g\left(u_{0}\right)^{2} / 2$ and zero, a less restrictive condition than (31). Solutions that satisfy (30) and (33) are referred to as edge solutions.

Additional constraint We have lost a small piece of information, because only the square of the flux of $v$ enters in the previous calculation. In order to guarantee that the flux has the correct sign as well as magnitude, we must have $v_{0} \leq v_{s}$ since $v$ must be decreasing across the transition layer. This gives an additional constraint on $u_{s}$ and $u_{0}$ :

$$
u_{s} \leq u_{0}
$$

in order for solutions to (30) and (31) or (33) to be relevant. 
2.5. Logistic growth. In this section we consider a variety of functional responses, in combination with logistic growth of the prey $f(u)=1-u$. The steady states for this model are therefore an extinction steady state, $(u=0, v=0)$, a prey-only steady state $(u=1, v=0)$, corresponding to primary succession before predators have arrived, and a coexistence steady state $\left.\left(u=u_{s}, v=v_{s}\right)\right)$. As stated previously, we also require the coexistence steady state to be stable, so have $g^{\prime}\left(u_{s}\right)<0$.

Logistic growth with type I predation We consider the case $h(u)=u$. Condition (30) for zero wave speed gives $u_{0}=0$ which prevents (31) or (33) from being satisfied. Thus zero wave speed solutions and edge solutions are not possible, irrespective of the behaviour of outer solutions. This confirms our linear analysis of wave speeds.

Logistic growth with type II predation With logistic growth and type II predation, $h(u)=(\alpha+1) u /(\alpha+u)$, we just have two parameters to consider, $\alpha$ and $\delta$. The coexistence steady state is stable if and only if $\delta>1-\alpha$. For a given $\alpha$ there exists a unique solution $u_{0}$ to (30), but no corresponding solutions to (31), so that stationary waves are not possible. This is in agreement with our linear analysis of wave speeds. It remains to consider the possibility of edge solutions.

Figure 5 shows the region in $\alpha-\delta$ parameter space where there are solutions to (30) and (33), subject to (34). Contours of constant values of $b$ are shown for $b=0.04$ and 0.08 , and the point where the maximum possible distance from the boundary, $b=0.118$, is attained. Decreasing $\gamma$ will increase $b$, but this is simply equivalent to a rescaling of space which stretches the domain.

It is straightforward to see from solutions to (25) that, for a transition layer at distance $b$ from the right-hand boundary,

$$
v(0)=\frac{v_{0}}{\cosh (\sqrt{\gamma \delta} b)} .
$$

Note that $v_{0}$ is independent of $\delta . v_{s}$ and $\sqrt{\gamma \delta} b$ are maximised by $\delta=1-\alpha$, and hence $v(0)$ is minimised by the same value of $\delta$. Figure 6 shows the ratio of $v(0)$ to $v_{s}$, which measures the maximum variation of $v$ across the domain $x \in(-\infty, 0]$. Thus $v$ can vary to no less than $75 \%$ of its steady state value across the whole domain. The variation from the transition layer at $x=-b$ to the boundary at $x=0$ is even smaller, at less than $1 \%$. These analytical predictions are verified by numerical simulations of the full system, indicated by the crosses in the figure. An example of such a numerically calculated edge solution is shown in Figure 7, for $\alpha=0.2$. As predicted by our analysis, the transition layer is less than 0.118 dimensionless space units away from the boundary.

To summarise, with logistic growth and type II predation, stationary waves are not possible, and edge solutions are possible in a restricted region of 


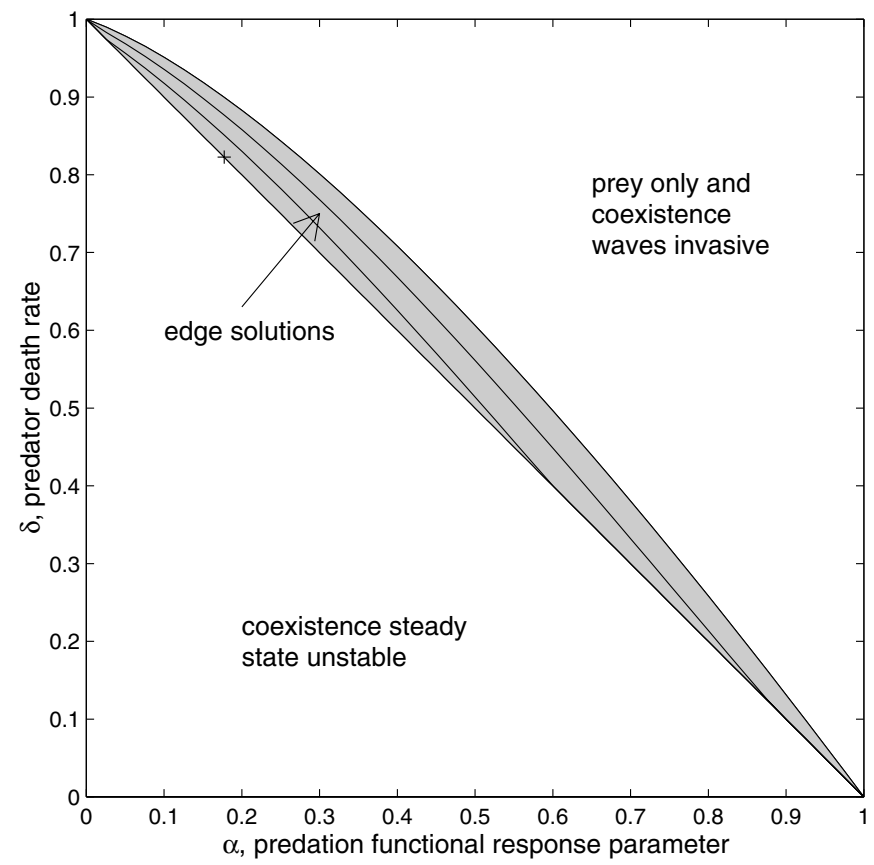

Figure 5. Characterisation of solution behaviour according to location in $\alpha-\delta$ parameter space, where $\alpha$ modulates the type II predation functional response, $h(u)=(\alpha+1) u /(\alpha+u)$, and $\delta$ is the predator death rate. In the shaded region, edge solutions are possible according to conditions (30), (33), and (34). The curves within the shaded region show contours of $b$, the distance of the transition layer from the right hand boundary, for $b=0.04$ and $b=0.08$. The cross indicates the location of the maximum distance from the boundary, $b=0.118$. For any given $\alpha$, the maximum distance $b$ is given by $\delta=1-\alpha$. 


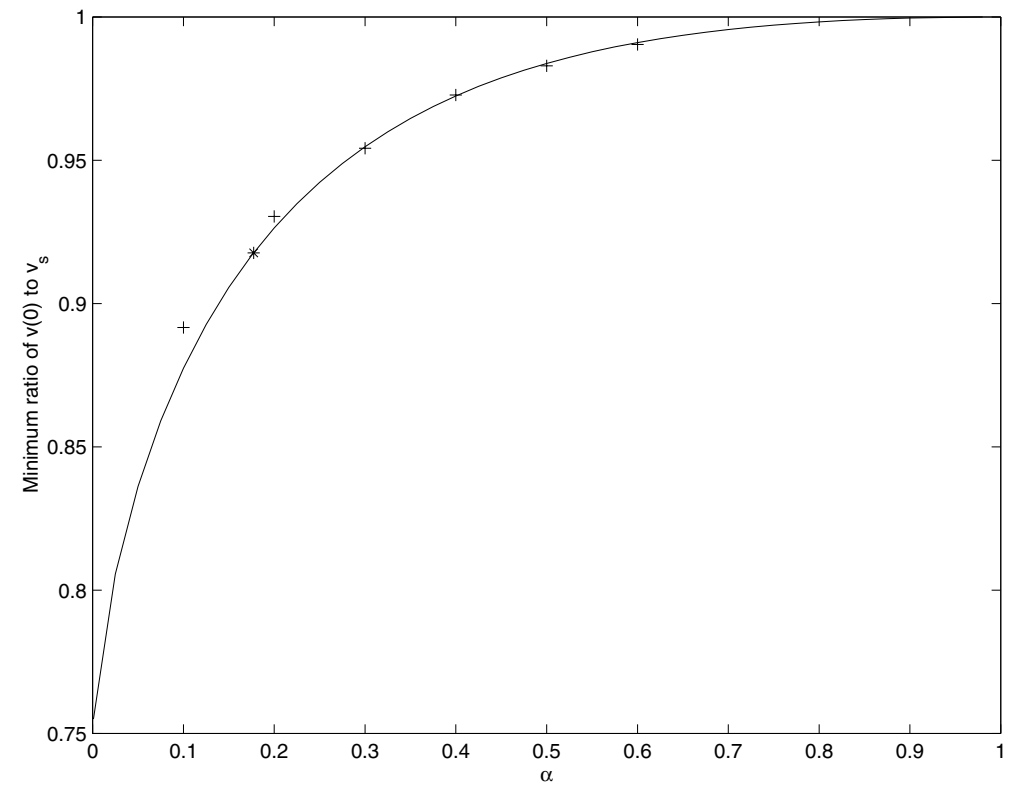

Figure 6. Maximum variation of predator density, $v$, across edge solutions for logistic growth and Type II predation, $h(u)=(\alpha+1) u /(\alpha+u)$. The panel shows the ratio of $v(0)$ to $v_{s}$, which has a minimum of 0.755 (to 3 s.f.). The asterisk indicates the value for the maximum distance from the boundary, $b=0.118$. The crosses show values calculated from numerical simulations of the full PDEs, which are clearly in very close agreement with our analysis. The parameters for those simulations were $\varepsilon=0.00001$, and $\delta=1-\alpha$, and an example simulation is illustrated in Figure 7 for $\alpha=0.2$. 

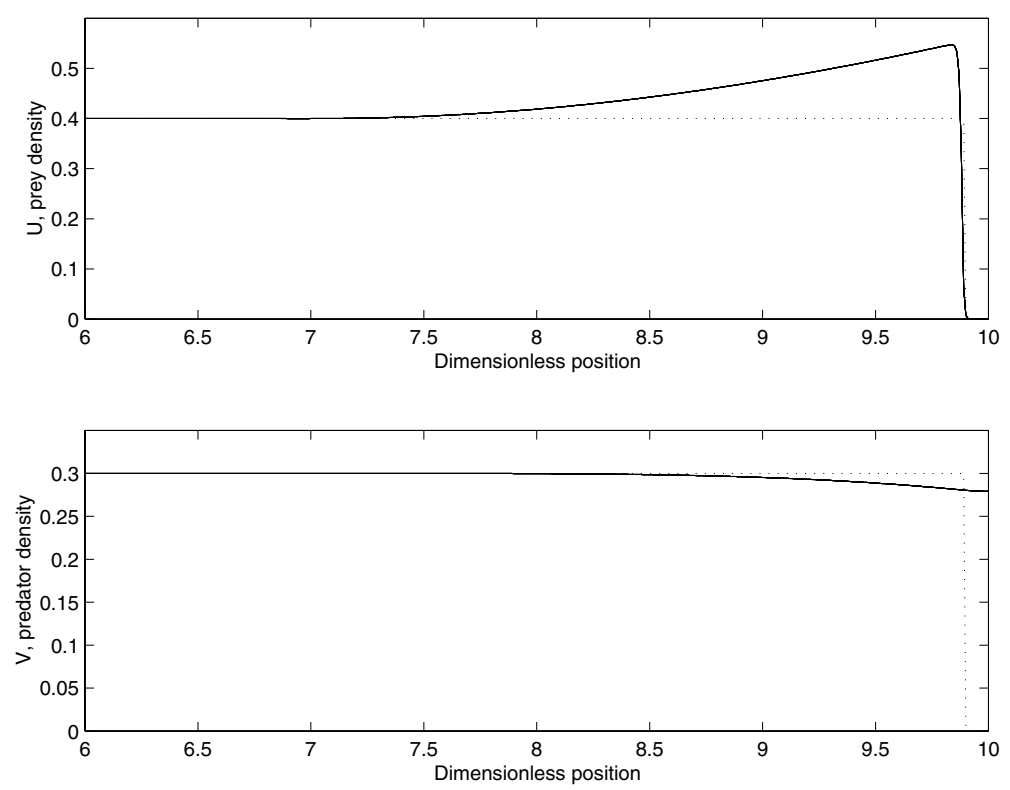

Figure 7. Edge solution calculated for logistic growth and Type II predation, $h(u)=(\alpha+1) u /(\alpha+u)$, with $\alpha=0.2, \delta=0.8$, and $\varepsilon=0.00001$. As predicted by our analysis, the transition layer is less than about 0.12 dimensionless space units away from the boundary, and $v$ varies very little across the whole domain.

parameter space. Such edge solutions are constrained to be close to the boundary, and can only support very limited variation in predator density. Furthermore, edge solutions are further restricted on finite, as opposed to semi-infinite, domains with zero-flux boundary conditions - edge solutions move closer to the boundary, and the variation in predator density is further reduced. Numerical investigations (not shown for brevity) also indicate that as finite domains become shorter, such edge solutions lose stability to the homogeneous coexistence steady state.

In contrast, we will see in the next section that with an Allee effect stationary waves and edge solutions are possible, and the presence of stationary solutions means that edge solutions can be arbitrarily far from the right hand boundary, with the full variation of predator density from $v_{s}$ down to zero.

2.6. Strong Allee effect. We consider the case where $f(u)$ is given by (12)-(13) and restrict consideration to a strong Allee effect $0<a<1$. Note that the prey-only wave moves forward for $a<0.5$, is stationary for $a=0.5$, and moves backwards for $0.5<a<1$ (14). In order for predators to be able to reverse the prey invasion we must find parameters $(a<0.5, \delta)$ such that the coexistence wave moves backwards. At the boundary of this region 
$a=a_{c}(\delta)$, the coexistence wave is stationary and satisfies (30)-(31). We calculate $u_{0}$ from (30) and use this to determine $a_{c}(\delta)$ from equation (31) (zero wave speed solution) or from equation (33) (edge solution). Results are given below.

Strong Allee effect with type I predation We first consider the case $h(u)=$ $u$. Figure 8 shows a division of $a-\delta$ parameter space according to solution type. There is a locus of points on which the coexistence wave speed is zero. To the left of this locus, coexistence waves are invasive, and to the right they are recessive. As discussed above, a second locus of points, at $a=0.5$, divides the space into the left part where colonising prey-only waves are invasive, and a right part where prey-only waves are recessive. Together then, three regions are defined, the left-most where both coexistence and prey-only are invasive; the centre region, where prey-only is invasive, but coexistence is recessive; and the right-most, where both coexistence and prey-only waves are recessive. Thus it is the centre region which is of interest. It corresponds to the case when an advancing wave of colonising prey is caught up by a wave of predators, but then the combined wave recedes, ultimately destroying both populations. Note that there is also a region in which the coexistence steady state is unstable.

Figure 9 shows the time evolution of spatial solutions which exhibit the predicted reversal behaviour - the parameters used are indicated on Figure 8 by the cross in the reversal region. Note that in the case illustrated, $\varepsilon$ is not actually that small, but our analytical predictions are remarkably accurate in such cases. Plotting these solutions in the $u-v$ phase plane (not shown for brevity) shows two heteroclinic connections which correspond to advancing and receding waves, and the receding wave does follow the $u$-nullclines as expected.

The parameter region for edge solutions given by (30), (33) is also shown on Figure 8 - the three crosses indicate the parameter values for numerically simulated examples which are illustrated in Figure 10. As parameters get closer to the locus of points for zero speed coexistence waves, edge solutions move away from the boundary. Stationary edge solutions are structurally stable in the sense that every point on the interior of this parameter region has a neighbourhood in $(a, \delta)$ space for which an edge solution also exists. By way of contrast, the zero wave speed solution does not have this propertyarbitrarily small changes in parameters will transform a stationary solution to an invasive or recessive wave. Hence we would not expect to see zero wave speed solutions in nature.

This analysis shows that for type I predation a high prey growth threshold is required for reversal, and intuitively this means that the prey-only wave must already be 'close' to reversal. This is also indicated by the proximity in parameter space of both zero speed loci. In fact, the minimum growth 


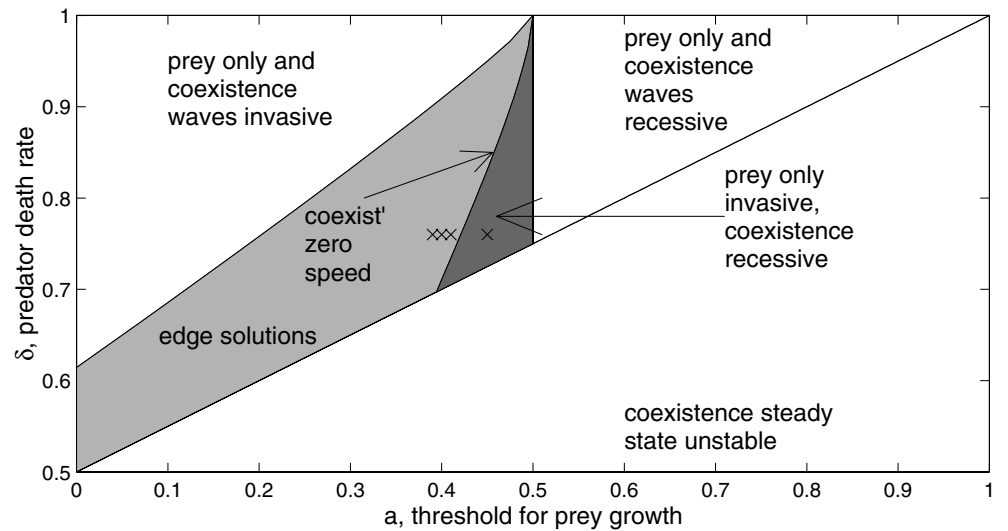

Figure 8. Characterisation of solution behaviour according to location in $a-\delta$ parameter space, where $a$ determines the Allee threshold for prey $(f(u)=4(u-$ $\left.a)(1-u) /(1-a)^{2}\right)$, and $\delta$ is the predator death rate. Zero wave speed is given by solving (30), (31) for $u_{0}, \delta$. Either side of the locus of zero wave speed parameter sets, waves travel in opposite directions. Thus the region shaded dark grey is where reversal is possible. The light grey region indicates where edge solutions are possible, as calculated by considering condition (33) in place of (31). The single cross shows the parameters for the reversal illustrated in Figure 9, and the three crosses indicate parameters for the edge solutions shown in Figure 10. 

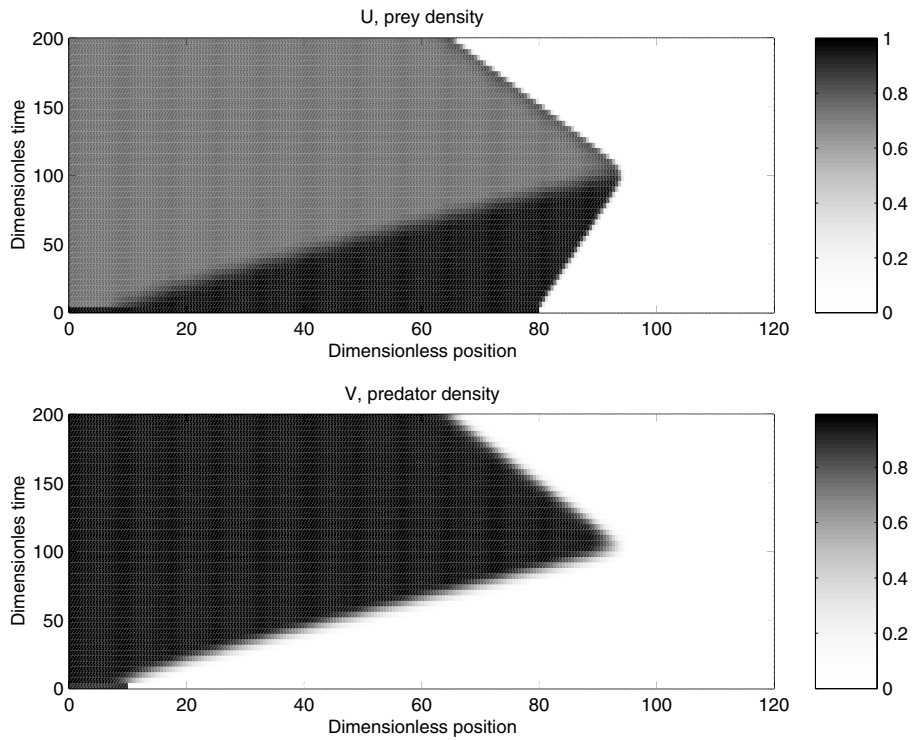

Figure 9. Spatial solution for the predator-prey model (3), with $f(u)=$ $4(u-a)(1-u) /(1-a)^{2}$. Parameters are $a=0.45, \delta=0.76, \gamma=1, \varepsilon=0.5$, corresponding to the cross in the reversal region of Figure 8 . The simulation shows the catch-up of predators, and subsequent reversal of the wave direction. Prey and predator densities are shown by a grey scale, with black being the maximum density, and white being zero. Thus we can see that the prey colonise vacant habitat slowly $\left(c_{u} \approx 0.15\right)$, while being caught up by the predators $\left(c_{v} \approx 0.9\right)$, but once caught, both prey and predators recede $\left(c_{u v} \approx-0.3\right)$. 

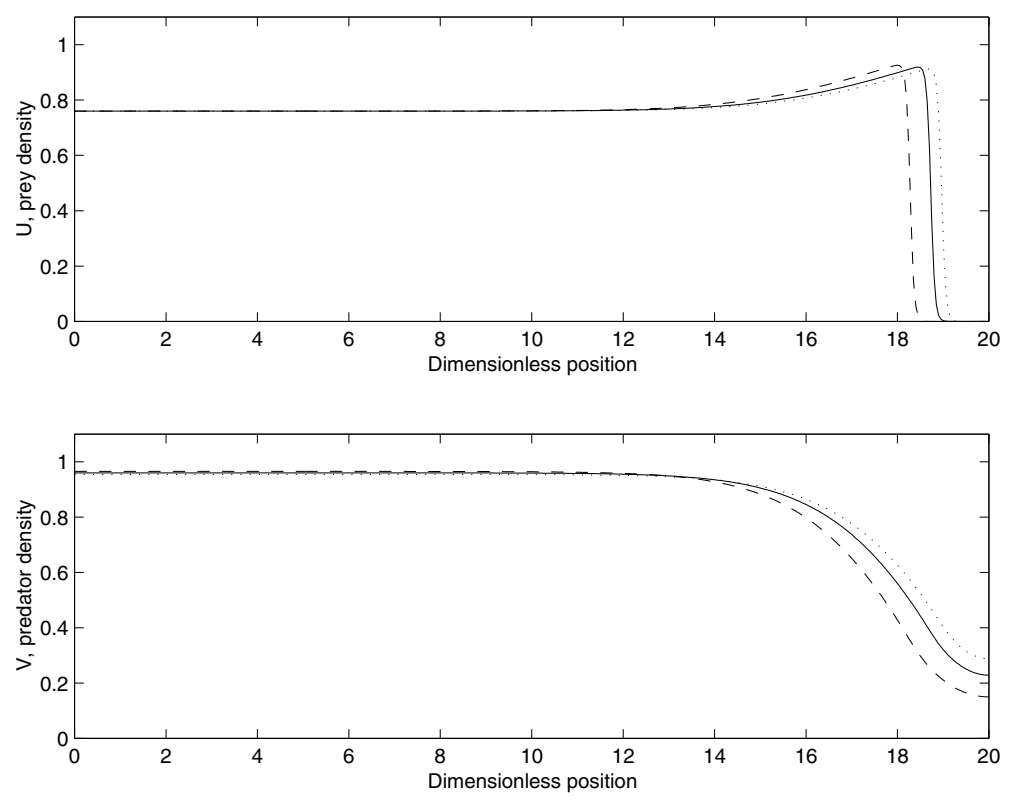

Figure 10. Edge solutions occur where predicted, for an Allee effect and type I predation. The solid line shows a stationary solution calculated for the same parameters as in Figure 2, with $a=0.4$. Increasing $a$ should move the edge solution away from the boundary, which is illustrated by the dashed line for $a=0.41$. Similarly, for $a=0.39$ (dotted line) the solution moves closer to the boundary. The parameters for these plots are indicated by crosses on Figure 8 . 
threshold allowing reversal, corresponding to the lower left point of the region shaded dark grey in Figure 8 , is $\min _{\delta} a_{c}(\delta) \approx 0.394$. Given that the prey carrying capacity is 1 , such a high threshold may not be ecologically plausible, and so in the following sections we explore the effect that different functional forms for $f$ and $h$ may have on the parameter ranges which allow reversal.

Strong Allee effect with types II and III predation We consider the effect of different functional responses on the 'reversibility' of prey invasion by predators. Here we consider whether type II and III predation can give reversal for a weaker, more biologically likely, Allee effect. We consider the cases with type II predation, $h(u)=(\alpha+1) u /(\alpha+u)$, and type III predation, $h(u)=\left(\alpha^{n}+1\right) u^{n} /\left(\alpha^{n}+u^{n}\right)$ where $n \geq 2$. The distinction between types II and III arises from the change in concavity in the type III functional response, which biologically describes predators switching from an alternate food source as prey density increases. In the type III case we consider $n=2$ and $n=3$.

Figure 11 shows the reversal region for type II predation whose boundary was calculated using (30)-(31). As the steepness of the functional response $\alpha$ increases the reversal region enlarges, and the minimum threshold for reversal diminishes. As $\alpha \rightarrow \infty$, we expect the reversal region to approach the simpler case of a type I functional response, since $\lim _{\alpha \rightarrow \infty} h(u)=u$. This is confirmed by following the minimum $a$ in the reversal region as $\alpha$ increases, and observing that it approaches 0.394, the minimum value in the type I case. This is illustrated in Figure 13 (solid line). Thus, type II predation is less likely to reverse an invasion than the simpler type I case.
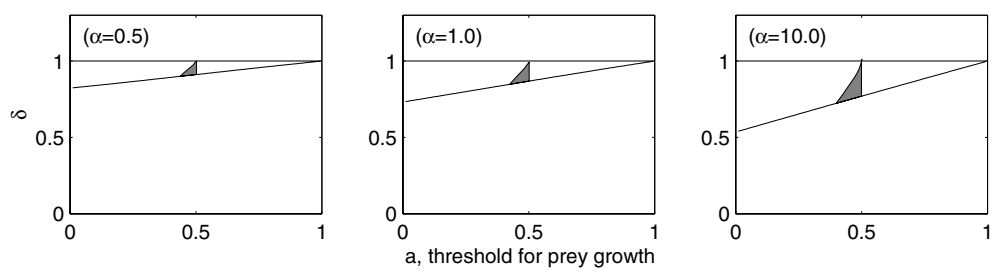

Figure 11. With type II predation, the solution behaviour is similar. $f(u)=$ $4(u-a)(1-u) /(1-a)^{2}, h(u)=(\alpha+1) u /(\alpha+u)$, and $\delta$ is the predator death rate. In the shaded region, prey-only and coexistence waves travel in opposite directions. As $\alpha$ increases, the reversal region extends to the left, but as $\alpha \rightarrow \infty$ it tends to a limit which is exactly that for the case of type I predation.

Now we consider the case with type III predation, $h(u)=\left(\alpha^{n}+1\right) u^{n} /\left(\alpha^{n}+\right.$ $\left.u^{n}\right)$. Figure 12 illustrates the reversal region for $n=2$ and $n=3$ as $\alpha$ 
increases. As before, increasing $\alpha$ allows the reversal region to extend to the left, but this time the limiting case is not identical to that for type I predation. Indeed, reversal is possible for smaller prey growth thresholds $a$ than either type I or type II predation.
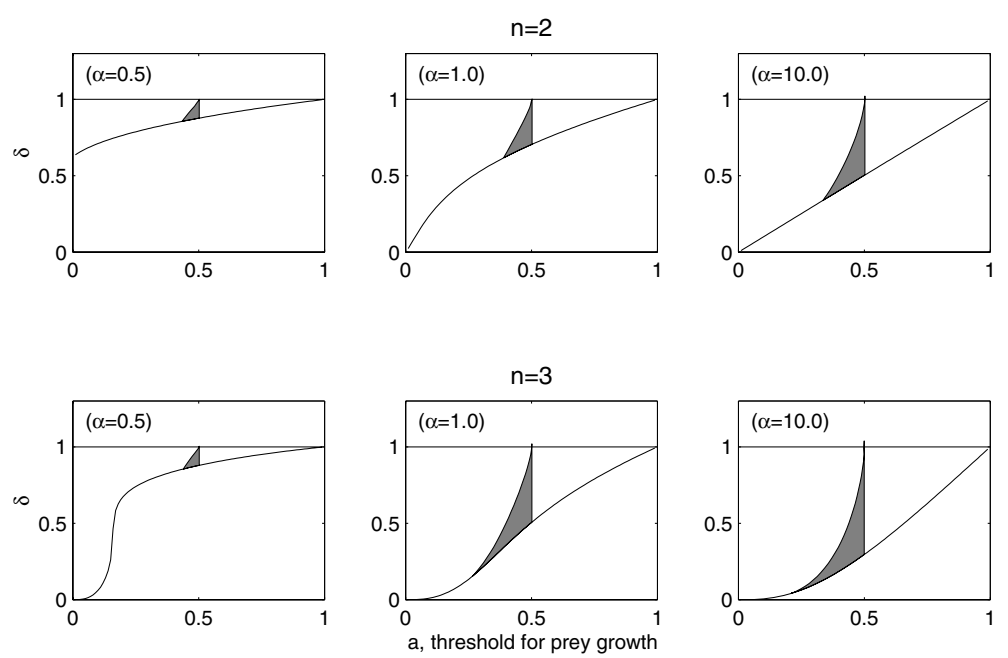

Figure 12. Characterisation of solution behaviour according to location in $a-\delta$ parameter space with a type III functional response, $h(u)=\left(1+\alpha^{n}\right) u^{n} /\left(\alpha^{n}+u^{n}\right)$. In the shaded regions, coexistence waves are recessive, whereas prey-only waves are invasive, so that the introduction of predators can lead to reversal. The reversal region gets larger as $\alpha$ increases.

Figure 13 illustrates the minimum prey growth threshold which allows reversal (i.e. the left most point of the reversal region), and shows how $n=2$ allows a lower threshold that for type II predation, and $n=3$ allows a still lower threshold. Thus, with $n=3$ we can get reversal with $a=0.3$, which is an ecologically more plausible threshold.

Figure 14 shows how the transition from invasive to recessive waves, in numerical simulations of the full system, occurs close to that predicted by our analysis. In the example illustrated, our analysis predicts reversal at $\delta \approx 0.133$, and we demonstrate reversal between $\delta=0.13$ and 0.14 (the other model details and parameters are in the figure legend).

\section{A patchy predator-prey model}

Predator-prey systems may not always be well suited to a continuum approach. The environment may be patchy, or movement may take place in discrete steps. The simplest form of movement has a population move from one patch to the next at a rate proportional to the difference in population in those patches. We consider one space dimension, with patches indexed 


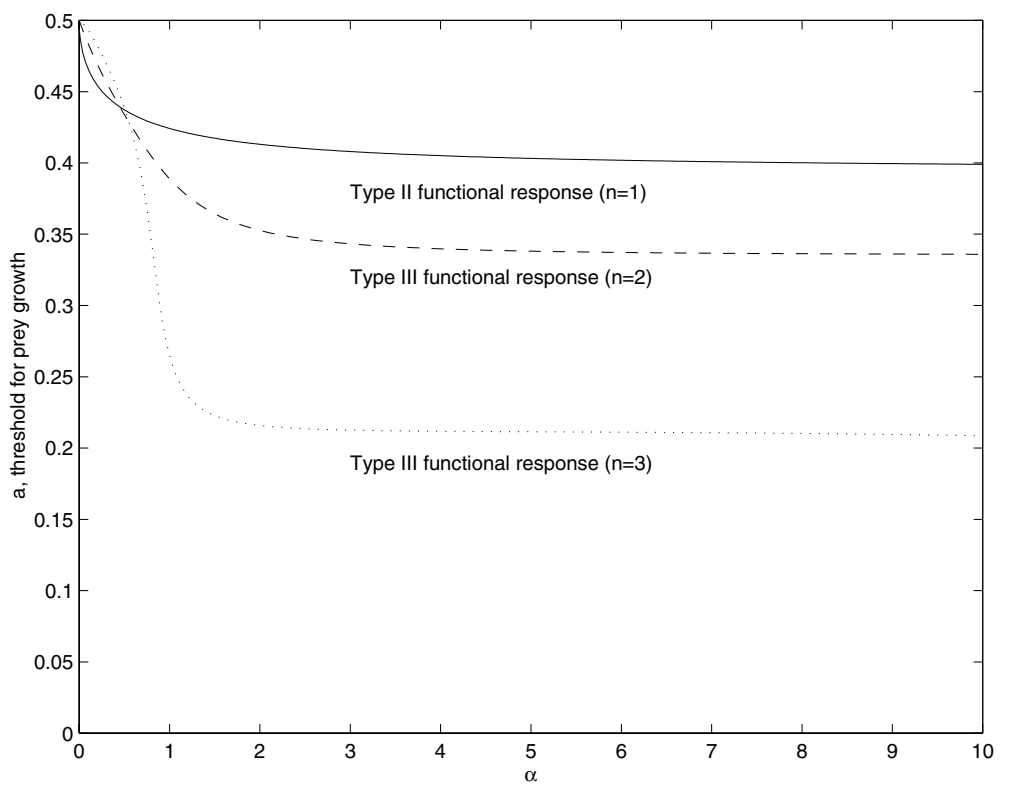

Figure 13. For an Allee effect and type II predation $(h(u)=\alpha u /(\alpha+u)$, solid line), as $\alpha$ varies, the range of $a$ which allows reversal changes, with the maximum range being reached as $\alpha \rightarrow \infty$. This maximum range is exactly that for the case with type I predation, since in the above limit, type II is equal to type I. For type III predation, $h(u)=\left(\alpha^{n}+1\right) u^{n} /\left(\alpha^{n}+u^{n}\right)$, smaller growth thresholds allow reversal (dashed and dotted lines). For example, with $n=3$ a threshold of $30 \%$ is possible. 

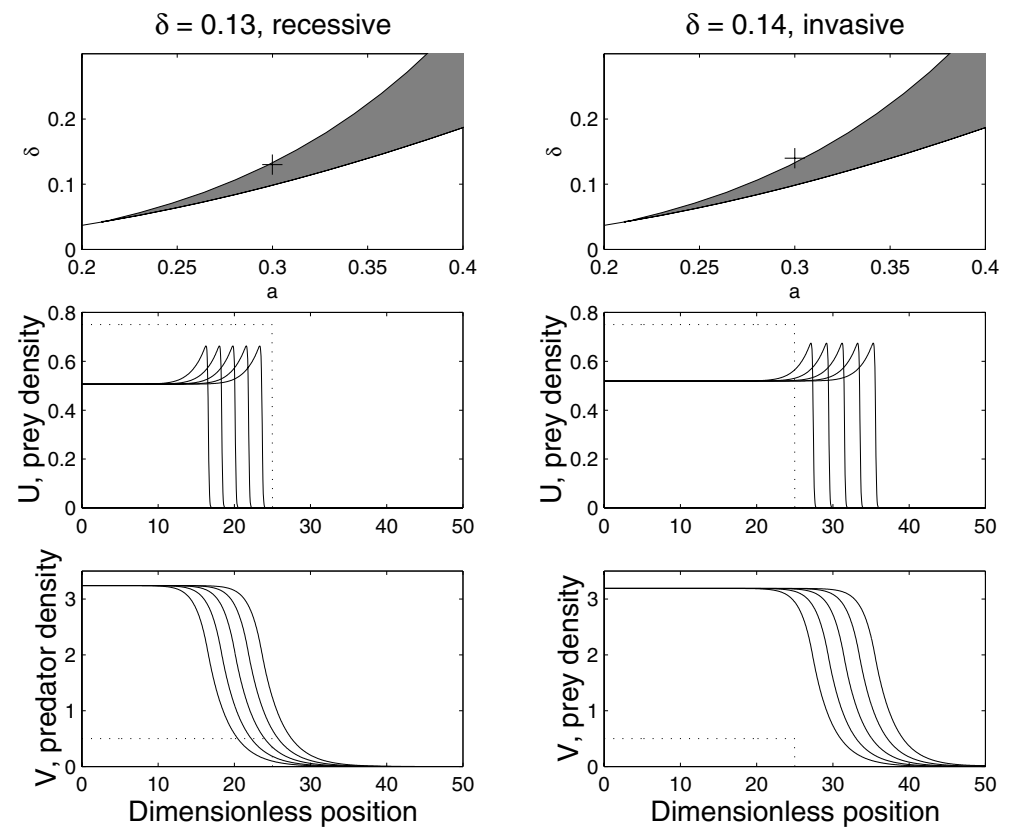

Figure 14. For type III predation, $h(u)=\left(\alpha^{3}+1\right) u^{3} /\left(\alpha^{3}+u^{3}\right)$, the change between invasive and recessive waves is very close to that at $\delta \approx 0.133$ predicted by theory. The top panels show the shaded reversal region and a cross indicating the parameters for the simulation below. Growth was $f(u)=4(u-a)(1-u) /(1-$ $a)^{2}$, with $a=0.3$, and the other parameters were $\varepsilon=0.01, \alpha=10, \gamma=1$. Initial conditions are shown by the dotted lines, and the solid lines show solutions from 1000 to 5000 dimensionless time units at intervals of 1000 . 
by $i$, so that

$$
\begin{aligned}
& u_{t}^{i}=u^{i}\left(f\left(u^{i}\right)-\frac{v^{i}}{u^{i}} h\left(u^{i}\right)\right)+\varepsilon d\left(u^{i-1}-2 u^{i}+u^{i+1}\right) \\
& v_{t}^{i}=\gamma v^{i}\left(h\left(u^{i}\right)-\delta\right)+d\left(v^{i-1}-2 v^{i}+v^{i+1}\right) .
\end{aligned}
$$

Thus the population can move to patch $i$ from patches $(i-1)$ and $(i+1)$, and does so according to the difference in population between the patches. If the distance between patches is $\Delta x$ and we scale $d=(\Delta x)^{-2}$ we regain our PDE model (3) as the patches strongly couple in the limit $\Delta x \rightarrow 0$. In this strong coupling limit, the patchy model will exhibit invasion and reversal under conditions identical to those for the PDE model.

We now consider the case where $d$ is finite, and deduce that, as with the PDE model (3), a strong Allee effect is needed to stop or reverse the wave.

3.1. Linear analysis. In the same way as for the continuous model we may look for travelling wave solutions $u^{i}(t)=u(i-c t)=u(z)$, and similarly for $v^{i}$. Then linearisation ahead of the coexistence wave, where $u^{i}=v^{i}=0$, determines a pair of uncoupled eigenvalue equations governing the decay of the solution ahead of the wave:

$$
\begin{array}{r}
c \lambda+f(0)+2 \varepsilon d(\cosh \lambda-1)=0 \\
c \lambda-\gamma \delta+2 d(\cosh \lambda-1)=0
\end{array}
$$

The second of these, (37), always gives real solutions for $\lambda$, for any value of the speed $c$, but equation (36) will give us conditions on $f$ which allow a zero speed wave, in the same way as for the continuous model.

If there were a zero speed wave, setting $c=0$ in the first equation, it follows that

$$
\cosh \lambda=\frac{2 \varepsilon d-f(0)}{2 \varepsilon d},
$$

and since for real $\lambda, \cosh \lambda \in[1, \infty)$, we need

$$
\frac{2 \varepsilon d-f(0)}{2 \varepsilon d} \geq 1
$$

which in turn leads to the requirement that $f(0) \leq 0$. Again we deduce that a 'strong' Allee effect is required for reversal. This is based on the requirement that solutions cannot be oscillatory as they approach $u=v=0$, since that would correspond to negative values of $u$ and $v$. Not only would it be irrelevant biologically to consider negative values, but it can be shown that non-negative initial data cannot evolve in such a way (see Lemmas 1 and 3 in the Appendix). 
3.2. Noninvasive solutions. In this section we will show that the discrete nature of patches means that, in the presence of a strong Allee effect, it is possible for for the coexistence travelling wave (7) to invade while the equivalent patchy formulation (35) does not. This may give rise to structurally stable stationary solutions for the predator-prey model (35) (see Figure 16 below). A similar phenomenon has been analysed by Keitt et al. (2000) in an ecological context for the prey-only model. It also arises as so-called 'propagation failure' in models for excitable systems in physiology (Keener, 1987, 1993).

Lemma 2 in the Appendix shows that if $u^{i}(0) \leq 1$ for all $i, u^{i}(t) \leq 1$ for all $t$ and $i$. If we consider this case $\left(u^{i} \leq 1\right)$ and a strong Allee effect for the prey $(f(0)<0)$, we can always find an $\varepsilon>0$ for which the solution to (35) is noninvasive.

Proposition 1. Assume that the minimum growth rate

$$
f_{-}=\min _{u \in[0,1]}\{u f(u)\}<0,
$$

and that this minimum occurs for some $u_{-} \in\left(0, u_{s}\right)$.

Consider initial conditions for the patchy model (35) with $0 \leq u^{i}(0) \leq 1$ and $v^{i}(0) \geq 0$ for all $i$, and suppose there exists $j$ such that $u^{i}(0) \leq u_{-}$for all $i \geq j$.

Then the solution to the patchy model (35) is noninvasive for all

$$
\varepsilon<\varepsilon_{0}=-\frac{f_{-}}{d\left(1-u_{-}\right)}
$$

Proof: We restate the above condition as $f_{-}+\varepsilon d\left(1-u_{-}\right)<0$.

If there is an invasive solution, then we must be able to define the earliest time $t^{*}$ that any patch $i \geq j$ crosses $u_{-}$:

$$
t^{*}=\inf \left\{t: u^{i}(t)>u_{-} \text {for some } i \geq j\right\} .
$$

Thus there exists a patch $k \geq j$ with $u^{k}\left(t^{*}\right)=u_{-}, u^{k+1}\left(t^{*}\right) \leq u_{-}$, and $u_{t}^{k}\left(t^{*}\right)>0$. From Lemmas $1-3,0 \leq u^{k-1}\left(t^{*}\right) \leq 1$, and $v^{k}\left(t^{*}\right) \geq 0$, so that

$$
\begin{aligned}
u_{t}^{k}\left(t^{*}\right) & =u_{-} f\left(u_{-}\right)-v^{k}\left(t^{*}\right) h\left(u_{-}\right)+\varepsilon d\left[u^{k-1}\left(t^{*}\right)-2 u_{-}+u^{k+1}\left(t^{*}\right)\right] \\
& \leq f_{-}+\varepsilon d\left(1-2 u_{-}+u_{-}\right)=f_{-}+\varepsilon d\left(1-u_{-}\right)<0
\end{aligned}
$$

which is a contradiction.

This bound is similar to, but tighter than, previously deduced bounds that ensure the prey-only system is stationary (Keitt et al., 2000). Figure 15 shows the above analytical bound, regions for invading and stationary solutions for the prey-only model (35) with $v \equiv 0$, and regions for invading, 
stationary and receding solutions for the full predator-prey system (35). Dynamics are strong Allee for the prey (12), and type I predation $h(u)=u$. The parameter range $0<a<0.5$ ensures that the prey-only PDE model would invade. The parameter $1 \leq d \leq 300$ describes the level of coupling between patches.

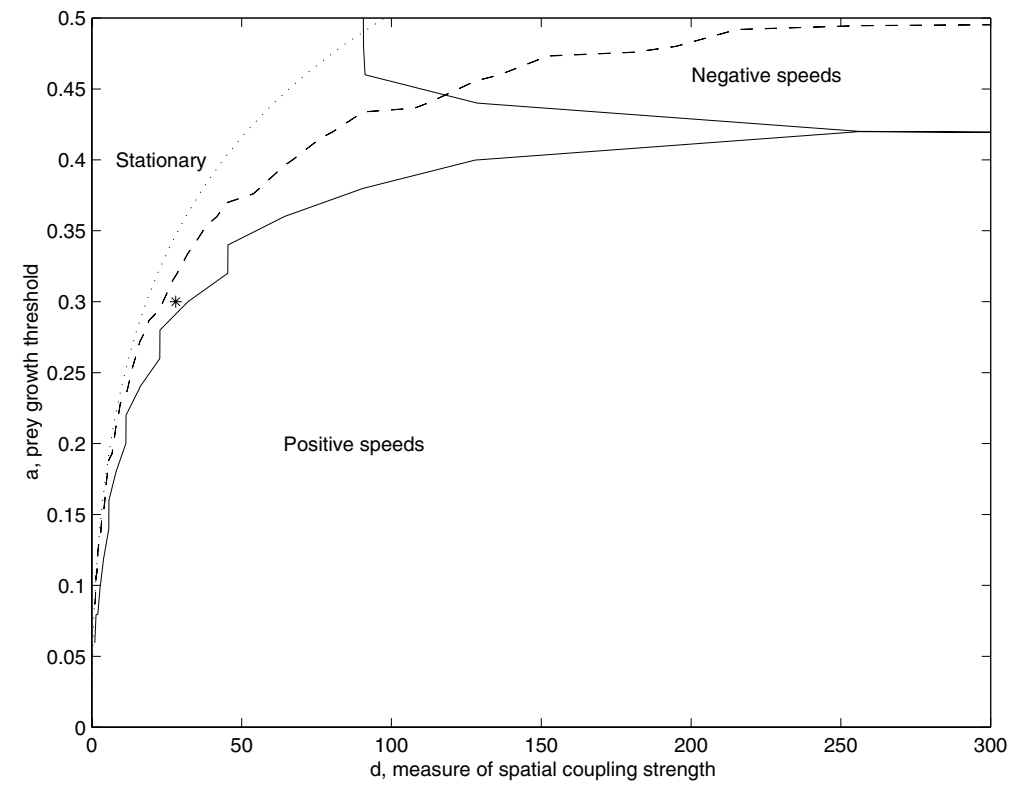

Figure 15. Contours for zero speeds for the patchy model (35), as the spatial coupling strength $d$ and the prey growth threshold $a$ vary. The dotted line is the analytical prediction for stationary fronts $\left(\varepsilon d<-f_{-} /\left(1-u_{-}\right)\right)$, the dashed line is the numerically calculated contour for pinned prey-only waves $(v \equiv 0)$, and the solid lines show zero-speed contours for the full predator-prey system. Note that this delimits regions with positive and negative speeds. The asterisk shows the parameters used for Figure 16, which according to this figure should give invading prey-only waves but stationary coexistence waves - this is indeed the case. Note that the contours are actually for speeds equal to \pm 0.001 .

Figure 16 illustrates this effect of switching from diffusive coupling to discrete patches. Here the coexistence wave for (3) invades whereas the coexistence wave for (35) stalls for sufficiently small spatial couplings. The simulation illustrated uses $d=28$ and $a=0.3$, corresponding to the asterisk in Figure 15, which is precisely in the region where prey-only waves invade but predator-prey coexistence waves are pinned. Further simulations, not shown for brevity, show that if $d$ is decreased, both waves are pinned from the outset, and if $d$ is increased, the coexistence wave does invade. The corresponding case of diffusive coupling was illustrated in Figure 3, with identical local dynamics - in that case the coexistence wave invaded. 

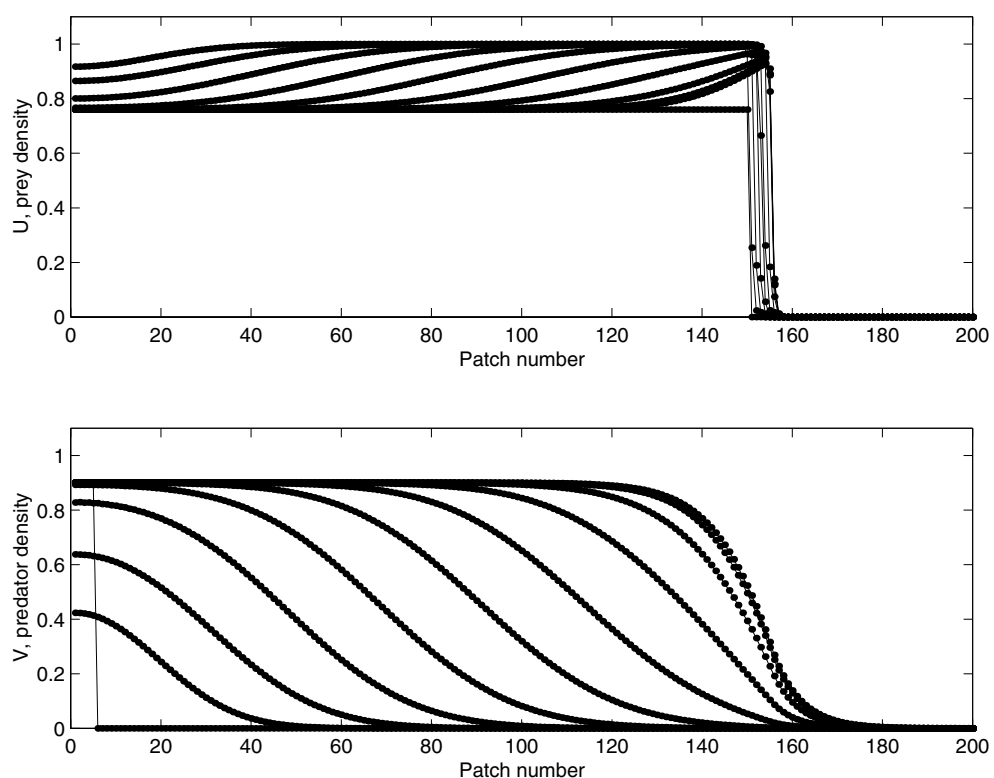

Figure 16. With a patchy model, stationary solutions are structurally stable. Other details such as functional response, prey growth, etc, are as in Figure 3, and the spatial coupling strength is $d=28$. This value corresponds to the asterisk in Figure 15, which is precisely in the region where prey-only waves invade but predator-prey coexistence waves are pinned. 


\section{Discussion}

Motivated by the suggestion that a recolonising lupin wave on Mount St. Helens may be slowed or reversed by the presence of herbivores, we have investigated whether such slowing or reversal is possible in general predatorprey systems, which are often used in models of plant-herbivore interactions.

Analysis of a general continuous model has shown that zero wave speed solutions are possible only with certain types of prey growth kinetics. We have identified a relatively simple set of integral conditions which can be used to determine whether reversal is possible for given model functions. The key requirement is for 'strong' Allee type growth functions, with a threshold effect, so that prey-only waves do move forwards, but are themselves close to reversal. Intuitively, the threshold effect means that when the integral whose sign determines the wave direction for the prey-only wave (the left hand side of equation (31) with $u_{0}=1$ ) is close to zero, the addition of predation (the left hand side of $(31)$ with $\left.u_{0}<1\right)$ can cause the net prey growth to become negative, hence reversing the wave. It is important to reinforce that just an Allee effect in which low densities give smaller but still positive growth rates cannot give reversal by predation; reversal requires that the prey must actually have negative growth rates at low densities. We have established that reversal is possible when prey growth thresholds are as low as $30 \%$ of prey carrying capacity, a biologically feasible threshold. In the presence of a weak Allee effect predators may slow, but not reverse, the spread of prey.

Even when zero speed waves on an infinite domain are not possible, fronts may stop when they approach a boundary with straightforward zero flux conditions. With logistic growth, reversal is not possible under any circumstances, but such edge solutions are possible. However, we have shown that such edge solutions permit very little variation in predator density, and that the transition layer is constrained to be very close to the boundary. These findings cast doubt on the relevance of this type of solution to the development of patchiness, as claimed by Hastings et al. (1997), unless the prey diffusion coefficient $\varepsilon$ is identically zero. In contrast, because an Allee effect allows reversal, it also allows edge solutions arbitrarily far from a boundary, and the full variation in predator density from its coexistence steady state level down to zero.

We have also shown that for a patchy model with the same type of predator-prey dynamics, non-invasive solutions are possible when prey movement is sufficiently weak, even when the continuum model predicts invasion. This explains why numerical simulations of continuous models using finite differences for spatial derivatives can yield spurious stationary solutionsthe system being solved in such cases may closely resemble the patchy model of Section 3. In fact, the numerical method which approximates (3) with (35) is referred to as the 'method of lines' numerical algorithm. 
These results naturally raise the question as to the existence of stationary and reversing waves in other types of model such as integrodifferential equations, coupled map lattices, and cellular automata. Another important type of dynamics to consider in future work is 'ratio dependent predation', where the predation rate does not just depend on how many prey are available, but also on how many predators are competing for that resource. Also, preliminary analysis shows that models with a generalist predator, which can survive on secondary prey species in the leading edge of the wave, more easily reverse prey invasions than the specialist predator models of this paper.

With particular reference to the original motivation for this study, namely the lupin-herbivore interaction at Mount St. Helens, there are a number of conclusions that may be drawn. Fagan and Bishop (2000) used a simple model incorporating exponential growth and diffusion of lupins to predict that the wave of recolonising lupins would reverse and the entire lupin population would become extinct. However, their study neglected the effect that declining lupin populations would have on the herbivores. If the herbivores are wholly dependent on lupins (i.e. the herbivores are not generalist predators, see above, or there are no alternative sources of food), then our analysis shows that this reversal could only occur if the lupins are subject to a strong Allee effect. The only caveats to our analysis within the reaction-diffusion framework are possibilities such as the ratio dependent functional response discussed above. Thus it is of considerable interest to determine whether lupins are indeed subject to an Allee effect.

It may be that the reaction-diffusion framework is inappropriate for the lupin-herbivore interaction at Mount St. Helens, and that the effects seen at the site are in part due to spatial and other interactions not included in the classical model framework. There is some evidence that herbivory is concentrated at the wave front (Fagan and Bishop, 2000). This may be because recently established lupin patches have not yet depleted the high levels of nutrients left over from the eruption, making the plants in those patches more 'tempting' to herbivores (B. Fagan, personal communication). Such an effect could require an age-structured approach to model the situation appropriately. Alternatively, integrodifference or integrodifferential equations may be the best way to capture the complexities of lupin seed dispersal, such as occasional long distance dispersal events which may lead to patchiness.

\section{ACKNOWLEDGEMENTS}

This work was supported in part by National Science Foundation grants DMS-9973212 (MAL) and DMS-9457816 (MRO and MAL) and in part by postdoctoral funds from the University of Utah (MRO). We are grateful to Bill Fagan and Alan Hastings for comments on the manuscript. 


\section{APPENDIX: Bounds on $u^{i}$ and $v^{i}$ for the patchy model}

In this section we construct bounds on $u$ and $v$ for the patchy model (35).

Lemma 1. If $v^{i}(0) \geq 0$ then $v^{i}(t) \geq 0$ for all $t>0$.

Proof: Suppose that $v^{i}<0$ at some time. Then let $t^{*}=\inf \left\{t: v^{i}(t)<\right.$ 0 for some $i$.

If follows that $v^{i}\left(t^{*}\right)=0, v^{i \pm 1}\left(t^{*}\right) \geq 0$, and $v_{t}^{i}\left(t^{*}\right)<0$. However, since $v^{i}\left(t^{*}\right)=0$, we have

$$
\begin{aligned}
v_{t}^{i}\left(t^{*}\right) & =\gamma v^{i}\left(t^{*}\right)\left[h\left(u^{i}\left(t^{*}\right)\right)-\delta\right]+d\left[v^{i-1}\left(t^{*}\right)-2 v^{i}\left(t^{*}\right)+v^{i+1}\left(t^{*}\right)\right] \\
& =0+d\left[v^{i-1}\left(t^{*}\right)-2 v^{i}\left(t^{*}\right)+v^{i+1}\left(t^{*}\right)\right] \geq 0
\end{aligned}
$$

This contradiction completes the proof.

Lemma 2. If $v^{i}(0) \geq 0$ and $u^{i}(0) \leq 1$ then $u^{i}(t) \leq 1$ for all $t>0$.

Proof: Suppose that $u^{i}>1$ at some time. Then let $t^{*}=\inf \left\{t: u^{i}(t)>\right.$ 1 for some $i$.

If follows that $u^{i}\left(t^{*}\right)=1, u^{i \pm 1}\left(t^{*}\right) \leq 1$, and $u_{t}^{i}\left(t^{*}\right)>0$. However, $f\left(u^{i}\left(t^{*}\right)\right)=$ $f(1)=0$, and by Lemma $1, v^{i}\left(t^{*}\right) \geq 0$, so that

$$
u_{t}^{i}\left(t^{*}\right)=-v^{i}\left(t^{*}\right) h\left(u^{i}\left(t^{*}\right)\right)+\varepsilon d\left[u^{i-1}\left(t^{*}\right)-2 u^{i}\left(t^{*}\right)+u^{i+1}\left(t^{*}\right)\right] \leq 0,
$$

which contradicts our assumption on $u_{t}^{i}\left(t^{*}\right)$.

Lemma 3. If $u^{i}(0) \geq 0$ then $u^{i}(t) \geq 0$ for all $t>0$.

Proof: Now suppose that $u^{i}<0$ at some time. Then let $t^{*}=\inf \left\{t: u^{i}(t)<\right.$ 0 for some $i$.

If follows that $u^{i}\left(t^{*}\right)=0, u^{i \pm 1}\left(t^{*}\right) \geq 0$, and $u_{t}^{i}\left(t^{*}\right)<0$. However, $h\left(u^{i}\left(t^{*}\right)\right)=$ $h(0)=0$, so that

$$
u_{t}^{i}\left(t^{*}\right)=\varepsilon d\left[u^{i-1}\left(t^{*}\right)-2 u^{i}\left(t^{*}\right)+u^{i+1}\left(t^{*}\right)\right] \geq 0,
$$

which contradicts our assumption on $u_{t}^{i}\left(t^{*}\right)$.

\section{References}

Aronson, D. G. and H. F. Weinberger (1975). Nonlinear diffusion in population genetics, combustion, and nerve pulse propagation. In J. A. Goldstein (Ed.), Lecture Notes in Mathematics, Volume 446, pp. 5-49. Berlin: Springer-Verlag.

Dunbar, S. R. (1986). Traveling waves in diffusive predator-prey equations: periodic orbits and point-to-periodic heteroclinic orbits. SIAM J. Appl. Math. 46, 10571078.

Fagan, W. F. and J. Bishop (2000). Trophic interactions during primary succession: Herbivores slow a plant reinvasion at Mount St. Helens. Am. Nat. 155, 238-251. 
Hadeler, K. P. and F. Rothe (1975). Travelling fronts in nonlinear diffusion equations. J. Math. Biol. 2, 251-263.

Hastings, A., S. Harrison, and K. McCann (1997). Unexpected spatial patterns in an insect outbreak match a predator diffusion model. Proc. R. Soc. Lond. B 264, $1837-1840$.

Hosono, Y. (1998). The minimal speed of traveling fronts for a diffusive Lotka Volterra competition model. Bull. Math. Biol. 60, 435-448.

Keener, J. P. (1987). Propagation failure in coupled systems of discrete excitable cells. SIAM J. Appl. Math. 47, 556-572.

Keener, J. P. (1993). The effects of discrete gap junction coupling on propagation in myocardium. J. Theor. Biol. 148, 49-82.

Keitt, T. H., M. A. Lewis, and R. D. Holt (2000). Allee effects, invasion pinning and species borders. Submitted to Am. Nat.

Lewis, M. A. and P. Kareiva (1993). Allee dynamics and the spread of invading organisms. Theor. Pop. Biol. 43, 141-158.

May, R. (1974). Model Ecosystems. Princeton NJ: Princeton University Press.

Murray, J. D. (1989). Mathematical Biology. Berlin: Springer Verlag.

Perko, L. (1991). Differential Equations and Dynamical Systems. New York: Springer Verlag.

Rothe, F. (1981). Convergence to pushed fronts. Rocky Mountain J. of Mathematics $11,617-633$.

Sherratt, J. A., B. T. Eagan, and M. A. Lewis (1997). Oscillations and chaos behind predator-prey invasion: Mathematical artefact or ecological reality? Phil. Trans. R. Soc. Lond. B 352, 21-38.

Smoller, J. (1994). Shock waves and reaction-diffusion equations. New York: Springer Verlag.

Weinberger, H. F., M. A. Lewis, and B. Li (2000b). Analysis of the linear conjecture for spread in cooperative models. Submitted to J. Math Biol.

Weinberger, H. F., M. A. Lewis, and B. Li (2000a). Spreading speeds and the linear conjecture for two-species competition models. Submitted to J. Math Biol. 